\title{
Amplitude Distribution for Radio Signals Reflected by Meteor Trails, II
}

Albert D. Wheelon

\author{
Contribution from Space Technology Laboratories, Inc. \\ P.O. Box 95001, Los Angeles, Calif.
}

(Received October 19, 1961; revised November 22, 1961)

\begin{abstract}
The bivariate probability distribution for two composite meteor signals displaced in time is derived theoretically using the Markoff statistical combination technique. Both the effects of numerous, small meteors and the residual reflections from infrequent large meteors are treated simultaneously. For the case of exponential decav of component signal spikes which are themselves distributed as the inverse square of their initial amplitudes, we find that the joint probability that a composite signal $R_{1}$ is observed at time $t_{1}$ and $R_{2}$ at $t_{2}$, seconds later, is given exactly by the following expression involving elliptic functions:
\end{abstract}

$$
\begin{aligned}
& W\left(R_{1}, R_{2}, \tau\right)=\frac{2}{\pi} \frac{\sigma^{2}\left(1-e^{-\tau / \eta}\right)}{\left(R_{1}^{2}+\sigma^{2}\right)^{3 / 2}} \cdot \frac{1}{\left[\sigma^{2}\left(1-e^{-\tau / \eta}\right)^{2}+\left(R_{2}-R_{1} e^{-\tau / \eta}\right)^{2}\right]} \\
& \frac{E\left[\sqrt{\frac{4 R_{1} R_{2} e^{-\tau / \eta}}{\sigma^{2}\left(1-e^{-\tau / \eta}\right)^{2}+\left(R_{2}+R_{1} e^{-\tau / \eta}\right)^{2}}}\right]}{\left[\sigma^{2}\left(1-e^{-\tau / \eta}\right)^{2}+\left(R_{2}+R_{1} e^{-\tau / \eta}\right)^{2}\right]^{1 / 2}}
\end{aligned}
$$

where $\sigma=Q_{\nu} \eta$ and $\nu$ is the average rate of occurrence of meteor signal spikes of all sizes and $\eta$ is the exponential decay time of each initial spike. This result reduces to the usual limiting forms in the case of $\tau$ very large or very small relative to the decay time $\eta$.

\section{Introduction}

The reflection of VHF radiowave signals from meteor trails in the E-region of the ionosphere is interesting for several reasons. It occurs both as radar backscattering from the trails and as propagation over oblique paths ranging out to $1,500 \mathrm{~km}$. The latter is significant because of its communication opportunities, and in that context it is important to know as much about the signal structure as possible. The composite meteor signal is the result of reflections from both large and small meteor trails, all in various stages of decay. The large meteors are easily recognized as individual signal spikes in amplitude versus time records. However, the far greater number of small, indistinguishable meteors also make an important contribution to the composite signal. The basic problem of understanding the signal structure is to treat the continuum of trail sizes simultaneously.

The probability distribution for the envelope of a meteor signal composed of reflection contributions from many trails of various sizes was derived theoretically in an earlier paper [Wheelon, 1960]. Both the effects of numerous, small meteors and the residual reflections from infrequent, large meteors were treated simultaneously. For the particular case of exponential decay of initial spikes which are themselves distributed as the inverse square of their amplitudes, we found that the probability that the composite signal amplitude should exceed a prescribed level $r$ is given by:

$$
P(R>r)=\frac{1}{\left[1+\frac{r^{2}}{(\nu \eta Q)^{2}}\right]^{1 / 2}},
$$

where $\nu$ is the average rate at which echoes of all sizes occur, $\eta$ is the characteristic decay time of each meteor spike and is set by the diffusive decay of the trail itself. Expression (1.1) behaves like a Rayleigh distribution for small amplitude margins $r$. For the larger, less likely signals $r$, it agrees with the result predicted by elementary analysis of isolated meteor reflections in various average states of decay. 
Essentially the same result was established by Bain [1960] in Britain and published almost simultaneously. His treatment goes to somewhat greater lengths to remove a potential divergence for small signal values by renormalizing the constant of proportionality $Q$ in the assumed distribution $\left(Q / p^{2}\right)$ for the strength of individual spikes $p$ in terms of an effective amplitude cutoff $\epsilon$. However, it would appear that this procedure is not required, since both (1.1) and the amplitude distribution corresponding thereto,

$$
W(R) d R=\frac{\nu \eta Q R d R}{\left[R^{2}+(\nu \eta Q)^{2}\right]^{3 / 2}},
$$

are well behaved for small signal amplitudes. It seems that the statistical combination of individual signals discriminates against the small signals, and that the weighted sum is insensitive to the error made in trying to extend the function $Q / p^{2}$ (which was curve-fitted to the experimental data) to smaller values of $p$.

In point of fact, it is the behavior of this assumed distribution for large $p$ which causes trouble, in the sense that (1.2) does not possess finite moments of any order. This is due to its behavior for exceptionally large signals,

$$
\lim _{R \rightarrow \infty} W \simeq \frac{\nu \eta Q}{R^{2}}
$$

which can, in turn, be traced to the mild behavior of $Q / p^{2}$ for large $p$.

The purpose of the present paper is to extend our knowledge of meteor signal structures by deriving the bivariate probability distribution for composite meteor signals displaced in time by an interval $\tau$. Such a result is evidently important in communication applications, since the time that a meteor scatter circuit is open is related to the interval during which all signals are above a specified threshold. The correlation of signal amplitudes between two instants is another measure of communication capacity. All of these basic features of a meteor circuit are derivable from the joint probability,

$$
W\left(R_{1}, R_{2}, \tau\right) d R_{1} d R_{2},
$$

that the composite signal assumes a precise value $R_{1}$ at time $t$, and becomes exactly $R_{2}$ at a time $t_{2}, \tau$ seconds later. The probability density $W\left(R_{1} R_{2} \tau\right)$ will be derived in this paper. A third paper in this series will use this result to calculate communication characteristics of meteor scatter circuits.

\section{General Expression for Bivariate Distribution}

As in the first paper of this series, we shall use the Markoff method [Chandrasekhar, 1943] to calculate the probability distribution for composite meteor signals. First, a word about notation and convention. Consider the typical signal history shown in figure 1, where time is run positively toward the past for analytical convenience. The two instants at which we wish to estimate the signal probability distribution are denoted by $t_{1}$ and $t_{2}$; although $t_{2}$ can and will later be chosen as the time origin. A large but finite interval $T$ is chosen in which $N$ meteor bursts are assumed to have occurred. $\quad N$ is a statistical quantity, whose mean value is $\nu T$, where $\nu$ denotes the average rate of meteor occurrence. The limit as $T$ goes to infinity will be taken later in the analysis, after convergence of certain integrals is assured.

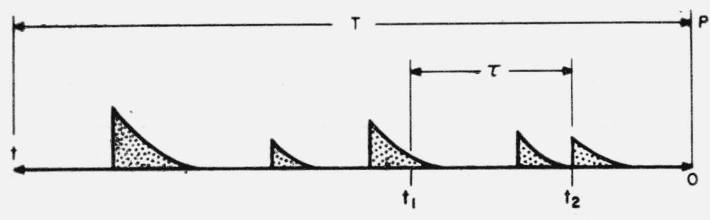

FIGURE 1. Typical succession of meteor spikes with initial amplitude $\mathrm{p}$, indicating measuring instants $\mathrm{t}_{1}$ and $\mathrm{t}_{2}$, separation time $\tau$, and total interval $\mathrm{T}$. 
The basic statistical problem is to calculate the probability density for observing composite signals $R_{1}$ and $R_{2}$ at $t_{1}$ and $t_{2}$ respectively. These total signals are compounded from the residual signal elements of all prior meteor spikes in the interval $T$. All signals received prior to $t_{2}$ contribute in some residual way to both $R_{1}$ and $R_{2}$. On the other hand, spikes received after $t_{2}$ but prior to $t_{1}$ contribute only to $R_{1}$. Of course, the individual spikes contribute to $R_{1}$ and $R_{2}$ in different measure, because of differential amplitude decay and phase relationships. However, one can write explicit expressions for the two total signals,

$$
\begin{aligned}
& \vec{R}_{1}=\sum_{i=1}^{N} \vec{p}_{i} \lambda\left(t_{i}-t_{1}\right) \\
& \vec{R}_{2}=\sum_{i=1}^{N} \vec{p}_{i} \lambda\left(t_{i}-t_{2}\right),
\end{aligned}
$$

in terms of the discontinuous function

$$
\lambda(t)= \begin{cases}e^{-t / \eta}, t>0 \\ 0, t<0\end{cases}
$$

which has been introduced for analytical convenience. This function allows one to write both $R_{1}$ and $R_{2}$ as formal summations over all $N$ initial spikes $\vec{p}_{i}$, yet ensures that those spikes received after the individual measuring events are not actually included. This is essentially a bookkeeping device, but is of considerable utility in organizing the subsequent analysis.

The joint probability distribution for $\vec{R}_{1}$ and $\vec{R}_{2}$ is given by Markoff's general method [Chandrasekhar, 1943] as a double Fourier transform of the characteristic function $B\left(k_{1}, k_{2}\right)$

where

$$
W\left(R_{1} R_{2}\right)=(2 \pi)^{-4} \int d^{2} k_{1} \int d^{2} k_{2} e^{i\left(\vec{k}_{1} \cdot \vec{R}_{1}+\vec{k}_{2} \cdot \vec{R}_{2}\right)} B\left(k_{1} k_{2}\right)
$$

$$
B\left(k_{1} k_{2}\right)=\left\langle\exp i\left[\overrightarrow{k_{1}} \cdot \sum_{i=1}^{N} \vec{p}_{i} \lambda\left(t_{i}-t_{1}\right)+\vec{k}_{2} \cdot \sum_{i=1}^{N} \vec{p}_{i} \lambda\left(t_{i}-t_{2}\right)\right]\right\rangle_{t_{1}, N} .
$$

The brackets indicate that one is to average over the three statistical features of the individual signals: (1) the distribution of initial pulse heights $p_{i}$ and their random phase, (2) the probability of finding a spike $\vec{p}_{i}$ at the instant $t_{i}$, and (3) the actual number of echoes $N$ in the fixed interval $T$. Because the individual meteors are statistically independent of one another (i.e., $i$ ), one can write

$$
B\left(k_{1} k_{2}\right)=\left\langle\left\langle\exp i\left[\overrightarrow{k_{1}} \cdot \vec{p} \lambda\left(t-t_{1}\right)+\overrightarrow{k_{2}} \cdot \vec{p} \lambda\left(t-t_{2}\right)\right]\right\rangle_{t, p}^{N}\right\rangle_{N} .
$$

Since the train of meteor echoes apparently forms a Markoff process (no sense of history) of small probability, one can argue that the probability of observing exactly $N$ spikes in the interval $T$ should follow a Poisson distribution.

$$
P(N / T)=\frac{(\nu T)^{N}}{N !} e^{-\nu T}
$$

The average over $N$ of (2.5) is computed thus:

$$
\begin{aligned}
& B\left(k_{1} k_{2}\right)=\sum_{N=0}^{\infty} \frac{(\nu T)^{N}}{N !} e^{-\nu T}\left\langle e^{\overrightarrow{i k_{1}} \cdot \overrightarrow{p \lambda}\left(t-t_{1}\right)+\overrightarrow{i k}_{2} \cdot \overrightarrow{p \lambda}\left(t-t_{2}\right)}\right\rangle_{t, p}^{N} \\
& =\exp -\nu T\left\{1-\left\langle e^{\overrightarrow{i k_{1} \cdot \vec{p} \lambda\left(t-t_{1}\right)}+\overrightarrow{i k}_{2} \cdot \overrightarrow{p \lambda}\left(t-t_{2}\right)}\right\rangle_{t, p}\right\} .
\end{aligned}
$$


One can write the remaining averages over $\vec{p}$ and $t$ in terms of the probability $\gamma(\vec{p}, t)$ that a single meteor echo occurs at time $t$ and produces a vector signal $\vec{p}$ in the receiver.

$$
\left\langle e^{\overrightarrow{i k_{1}} \cdot \vec{p} \lambda\left(t-t_{1}\right)+\overrightarrow{i k}_{2} \cdot \overrightarrow{p \lambda\left(t-t_{2}\right)}}\right\rangle_{t, p}=\int d^{2} p \int_{0}^{T} d t \gamma(p, t) e^{\overrightarrow{i k_{1}} \cdot \overrightarrow{p \lambda}\left(t-t_{1}\right)+\overrightarrow{i k}_{2} \cdot \overrightarrow{p \lambda\left(t-t_{2}\right)}}
$$

Since the individual meteor echoes can occur with equal probability anywhere in the interval $T$,

$$
\gamma(\vec{p}, t)=\frac{1}{T} \gamma(\vec{p})
$$

The initial echo spikes $\vec{p}$ are randomly phased, since the distance from the transmitter to the individual meteor trails is a completely random variable. Hence,

$$
\int d^{2} p \gamma(\vec{p})=\frac{1}{2 \pi} \int_{0}^{\infty} d p D(p) \int_{0}^{2 \pi} d \theta
$$

where $\theta$ is an arbitrary phase reference, which we take to be the angle between $\vec{p}$ and $\vec{k}_{1}$. $D(p)$ represents the distribution of initial pulse heights. If $\phi$ denotes the angle between $\vec{k}_{1}$ and $\vec{k}_{2}$, one can use the equivalence (2.8) and the above to rewrite expression (2.7) for $B\left(k_{1} k_{2}\right)$ as follows:

$$
B\left(k_{1} k_{2}\right)=\exp -\frac{\nu}{2 \pi} \int_{0}^{\infty} d p D(p) \int_{0}^{2 \pi} d \theta \int_{0}^{T} d t\left[1-e^{i k_{1} p \cos \theta \lambda\left(t-t_{1}\right)} e^{i k_{2} p \cos (\theta+\phi) \lambda\left(t-t_{2}\right)}\right]
$$

The angular integration can be done using the standard formula:

$$
\int_{0}^{2 \pi} d \theta e^{i(a \sin \theta+b \cos \theta)}=2 \pi J_{0}\left(\sqrt{a^{2}+b^{2}}\right)
$$

giving:

$B\left(k_{1}, k_{2}, \phi\right)=\exp -\nu \int_{0}^{\infty} d p D(p) \int_{0}^{\infty} d t\left\{1-J_{0}\left(p \sqrt{\left[k_{1} \lambda\left(t-t_{1}\right)+k_{2} \cos \phi \lambda\left(t-t_{2}\right)\right]^{2}+\left[k_{2} \sin \phi \lambda\left(t-t_{2}\right)\right]^{2}}\right)\right\}$

where we have taken the limit of $T$ going to infinity, since the difference quantity in braces is now finite at the upper limit.

To make further progress, one must divide the time interval and introduce explicit expression (2.2) for the discontinuous time functions $\lambda\left(t-t_{1}\right)$ and $\lambda\left(t-t_{2}\right)$. At this point in the calculation, it is convenient to choose $t_{1}=0$ and $t_{2}=\tau$, since only the time difference $\left(t_{1}-t_{2}\right)=\tau$ is relevant to a stationary problem. Dividing the time interval into two segments: $0<t<\tau$ and $\tau<t<\infty$, allows one to write equation (2.11) out as follows:

$$
\begin{aligned}
B\left(k_{1}, k_{2}, \phi\right)=\exp -\nu \int_{0}^{\infty} d p D(p)\left\{\int_{0}^{\tau} d t\right. & {\left[1-J_{0}\left(k_{2} p e^{-t / \eta}\right)\right] } \\
& \left.+\int_{\tau}^{\infty} d t\left[1-J_{0}\left(p e^{-t / \eta} \sqrt{k_{2}^{2}+2 k_{2} k_{1} \cos \phi e^{\tau / \eta}+k_{1}^{2} e^{2 \tau / \eta}}\right)\right]\right\}
\end{aligned}
$$

\section{Distribution for Inverse Square Law}

The remaining integrals can be done in closed form if the distribution of initial pulse heights is assumed to have the form,

$$
D(p)=\frac{Q}{p^{2}} .
$$


This expression is more than an analytical convenience, in that experimental data on the distribution of individual pulse heights is well fitted by eq (2.13). The major assumption involved in using (2.13) is that the same law extends down to the small meteors which cannot be distinguished as individual spikes. This assumed distribution of initial pulse heights is not really an acceptable form in that the normalization integral

$$
\int_{0}^{\infty} d p D(p)=Q \int_{0}^{\infty} \frac{d p}{p^{2}}
$$

diverges at the lower limit, rather than approaching unity. Strictly speaking, this implies that the average rate of meteor signal occurrence $\nu$ is infinite, which, in turn, invalidates the assumption of the Poisson distribution (2.6). The cutoff procedure introduced by Bain [1960] remedies this deficiency in a formal way. However, the divergence of this law for small $p$ does not affect the final composite signal, as noted earlier. Its relatively slow decrease for the very large, exceptional rare meteors causes the real trouble.

The double integrations remaining in the evaluation of this characteristic function $B$ $\left(k_{1} k_{2}\right)$ in eq $(2.12)$ can be done by interchanging the order of $p$ and $t$ integration and setting

and

$$
u=p e^{-t / \eta} k_{2}
$$

$$
v=p e^{-t / \eta} \sqrt{k_{2}^{2}+2 k_{2} k_{1} \cos \phi e^{\tau / \eta}+k_{1}^{2} e^{2 \tau / \eta}}
$$

in the first and second integral, respectively. This gives for (2.12) the following:

$$
\begin{aligned}
& B\left(k_{1}, k_{2}, \phi\right)=\exp -\nu Q\left\{k_{2} \int_{0}^{\tau} d t e^{-t / \eta} \int_{0}^{\infty} \frac{d u}{u^{2}}\left[1-J_{0}(u)\right]\right. \\
& \left.\quad+\sqrt{k_{2}^{2}+2 k_{2} k_{1} \cos \phi e^{\tau / \eta}+k_{1}^{2} e^{2 \tau / \eta}} \cdot \int_{\tau}^{\infty} d t e^{-t / \eta} \int_{0}^{\infty} \frac{d v}{v^{2}}\left[1-J_{0}(v)\right]\right\} .
\end{aligned}
$$

The integrations are now uncoupled and can be done by noting that

$$
\int_{0}^{\infty} \frac{d x}{x^{2}}\left[1-J_{0}(x)\right]=1
$$

The final expression for the characteristic function becomes,

$$
B\left(k_{1}, k_{2}, \phi\right)=\exp -\nu \eta Q\left[k_{2}\left(1-e^{-\tau / \eta}\right)+\sqrt{k_{2}^{2} e^{-2 \tau / \eta}+2 k_{2} k_{1} \cos \phi e^{-\tau / \eta}+k_{1}^{2}}\right] .
$$

The bivariate probability density is calculated from this expression as the double Fourier transform of eq (2.3)

$$
W\left(\vec{R}_{1} \vec{R}_{2}\right)=\frac{1}{(2 \pi)^{4}} \int d^{2} k_{1} \int d^{2} k_{2} e^{i\left(\vec{k}_{1} \cdot \vec{R}_{1}+\vec{k}_{2} \cdot \vec{R}_{2}\right)} e^{-\sigma k_{2}\left(1-e^{-\tau / \eta)}\right.} e^{-\sigma \sqrt{k_{1}^{2}+2 k_{1} k_{2} \cos \phi e^{-\tau / \eta}+k_{2}^{2} e^{-2 \tau / \eta}}}
$$

where for notational convenience we have now set

$$
\sigma=\nu \eta Q .
$$

The integrals in eq (3.6) can be performed most readily if one makes the following linear vector transformation:

so that

$$
\vec{l}=\vec{k}_{1}+\vec{k}_{2} e^{-\tau / \eta},
$$

$$
W\left(\vec{R}_{1} \vec{R}_{2}\right)=(2 \pi)^{-4} \int d^{2} k_{2} \int d^{2} l e^{\overrightarrow{i l} \cdot \vec{R}_{1}} e^{\overrightarrow{i k}_{2} \cdot\left(\vec{R}_{2}-\vec{R}_{1} e^{-\tau / \eta)}\right.} e^{-\sigma k_{2}\left(1-e^{-\tau / \eta)}\right.} e^{-\sigma l}
$$

The $l$ and $k_{1}$ integrals are now separated and can be done analytically by using the intra-vector angular definitions exhibited in figure 2. With these conventions, one can write out the fourfold integration above as angular definitions for signal $\left(\vec{R}_{1}\right)$ and transform $\left(\vec{k}_{1}\right)$ vectors, all 


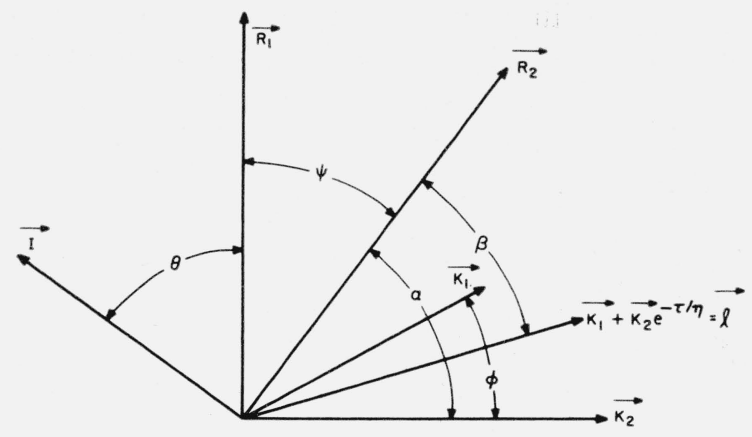

Figure 2. Intra-vector angular definitions.

with respect to an arbitrary phase reference vector $\vec{I}$.

$$
W\left(R_{1}, R_{2}, \psi, \theta\right)=\frac{1}{(2 \pi)^{4}} \int_{0}^{\infty} d l l e^{-\sigma l} \int_{0}^{2 \pi} d \beta e^{i l R_{1} \cos (\beta+\psi)} \int_{0}^{\infty} d k_{2} k_{2} e^{-\sigma k_{2}} \int_{0}^{2 \pi} d \alpha e^{i k_{2}\left[R_{2} \cos \alpha-R_{1} e^{-\tau / \eta} \cos (\alpha+\psi)\right]} .
$$

Using the integral result (2.10), we find the final expression for the bivariate vector probability distribution to be

$$
W\left(R_{1}, R_{2}, \psi, \theta\right)=\frac{\sigma^{2}\left(1-e^{-\tau / \eta}\right)}{(2 \pi)^{2}\left(R_{1}^{2}+\sigma^{2}\right)^{3 / 2}\left[\sigma^{2}\left(1-e^{-\tau / \eta}\right)^{2}+R_{1}^{2} e^{-2 \tau / \eta}-2 R_{1} R_{2} \cos \psi e^{-\tau / \eta}+R_{2}^{2}\right]^{3 / 2}} .
$$

One can verify that this expression is normalized to unity by integrating over the components of $\vec{R}_{1}$ and $\vec{R}_{2}$.

$$
\begin{aligned}
& \int_{0}^{\infty} d R_{1} R_{2} \int_{0}^{\infty} d R_{2} R_{2} \int_{0}^{2 \pi} d \psi \int_{0}^{2 \pi} d \theta W\left(R_{1}, R_{2}, \psi, \theta\right) \\
&=\frac{1}{2 \pi} \sigma^{2}\left(1-e^{-\tau / \eta}\right) \int_{0}^{\infty} \frac{d R_{1} R_{1}}{\left(R_{1}^{2}+\sigma^{2}\right)^{3 / 2}} \int_{0}^{2 \pi} d \psi \int_{0}^{\infty} d R_{2} R_{2} \\
& \\
& \quad \frac{1}{\left[R_{2}^{2}-2 R_{2} R_{1} e^{-\tau / \eta} \cos \psi+R_{1}^{2} e^{-2 \tau / \eta}+\sigma^{2}\left(1-e^{-\tau / \eta}\right)^{2}\right]^{3 / 2}} \\
&= \frac{1}{2 \pi} \sigma^{2}\left(1-e^{-\tau / \eta}\right) \int_{0}^{\infty} \frac{d R_{1} R_{1}}{\left(R_{1}^{2}+\sigma^{2}\right)^{3 / 2}} \int_{0}^{2 \pi} \frac{d \psi}{\left[-R_{1} e^{-\tau / \eta} \cos \psi+\sqrt{R_{1}^{2} e^{-\tau / \eta}+\sigma^{2}\left(1-e^{-\tau / \eta}\right)^{2}}\right]} \\
&= \frac{1}{2 \pi} \sigma^{2}\left(1-e^{-\tau / \eta}\right) \int_{0}^{\infty} \frac{d R_{1} R_{1}}{\left(R_{1}^{2}+\sigma^{2}\right)^{3 / 2}} \cdot \frac{2 \pi}{\sigma\left(1-e^{-\tau / \eta}\right)} \\
&= \int_{0}^{\infty} \frac{d x x}{\left(1+x^{2}\right)^{3 / 2}}=1 \mathrm{QED} .
\end{aligned}
$$

The distribution of signal amplitudes is obtained from (3.8) by integrating over the phase angles $\psi$ and $\theta$, and leads to the following expression involving complete elliptic functions.

$$
W\left(R_{1} R_{2}\right)=\frac{2}{\pi} \frac{\sigma^{2}\left(1-e^{-\tau / \eta}\right)}{\left(R_{1}+\sigma^{2}\right)^{3 / 2}} \cdot \frac{1}{\left[\sigma^{2}\left(1-e^{-\tau / \eta}\right)^{2}+\left(R_{2}-R_{1} e^{-\tau / \eta}\right)^{2}\right]} \frac{E\left[\sqrt{\frac{4 R_{1} R_{2} e^{-\tau / \eta}}{\sigma^{2}\left(1-e^{-\tau / \eta}\right)^{2}+\left(R_{2}+R_{1} e^{-\tau / \eta}\right)^{2}}}\right]}{\left[\sigma^{2}\left(1-e^{-\tau / \eta}\right)^{2}+\left(R_{2}+R_{1} e^{-\tau / \eta}\right)^{2}\right]^{1 / 2}} .
$$

The expected limiting forms of this result emerge if one assigns special values to the time displacement $\tau$. For example, if the time difference between the two measuring instants is large compared to the decay time of the individual signal specified $\eta$, one has

$$
\lim _{\tau \rightarrow \infty} W\left(R_{1} R_{2}\right)=\frac{2}{\pi} \frac{\sigma^{2}}{\left(R_{1}^{2}+\sigma^{2}\right)^{3 / 2}} \frac{1}{\left(R_{2}^{2}+\sigma^{2}\right)} \cdot \frac{E(0)}{\left(\sigma^{2}+R_{2}^{2}\right)^{1 / 2}}=\frac{\sigma}{\left(R_{1}^{2}+\sigma^{2}\right)^{3 / 2}} \cdot \frac{\sigma}{\left(R_{2}^{2}+\sigma^{2}\right)^{3 / 2}} .
$$


This is just the product of the individual distributions (see eq 1.2) for $R_{1}$ and $R_{2}$ derived in the first paper of this series [Wheelon, 1960]. The opposite extreme is somewhat more subtle, but can be extracted by taking the limit as $\tau$ goes to zero.

$$
\lim _{\tau \rightarrow 0} W\left(R_{1} R_{2}\right)=\frac{2}{\pi} \frac{\sigma}{\left(R_{1}^{2}+\sigma^{2}\right)^{3 / 2}} \frac{E\left[\sqrt{\frac{4 R_{1} R_{2}}{\left(R_{1}+R_{2}\right)^{2}}}\right]}{\sqrt{\left(R_{1}+R_{2}\right)^{2}}} \cdot \lim _{\tau \rightarrow 0}\left[\frac{\tau \frac{\sigma}{\eta}}{\left(\frac{\tau \sigma}{\eta}\right)^{2}+\left(R_{1}-R_{2}\right)^{2}}\right] .
$$

Using the following limit definition of the Dirac Delta function,

we have

$$
\delta(x)=\frac{1}{\pi} \lim _{\epsilon \rightarrow 0}\left(\frac{\epsilon}{\epsilon^{2}+x^{2}}\right)
$$

$$
\begin{aligned}
\lim _{\tau \rightarrow 0} W\left(R_{1} R_{2}\right) & \left.=\frac{2 \sigma}{\left(R_{1}^{2}+\sigma^{2}\right)^{3 / 2}} \cdot \frac{E\left[\sqrt{\frac{4 R_{1} R_{2}}{\left(R_{1}+R_{2}\right)^{2}}}\right]}{\left(R_{1}+R_{2}\right)}\right]_{\delta\left(R_{1}-R_{2}\right)} \\
& =\frac{\sigma}{\left(R_{1}^{2}+\sigma^{2}\right)^{3 / 2}} \cdot \frac{\delta\left(R_{1}-R_{2}\right)}{R_{1}} .
\end{aligned}
$$

This indicates that as the time displacement goes to zero, the two signals should coalesce as prescribed by the Delta function and their distribution be described by the previous result for the single time expression. Note that the factor $R_{1}$ in the Jacobian for polar coordinates is just cancelled by the denominator term.

The author acknowledges several valuable discussions of this paper with Dr. T. A. Magness.

\section{References}

Bain, W. C., The received-amplitude distribution produced by radio sources of random occurrence and phase, Proc. Inst. Elec. Engrs., Part C, Mono. 389E (July 1960).

Chandrasekhar, S., Stochastic problems in physics and astronomy, Rev. Mod. Phys. 15, 1 (1943).

Wheelon, Albert D., Amplitude distribution for radio signals reflected by meteor trails I, J. Research NBS

64D (Radio Prop.), 449 (1960).

(Paper 66D3-192) 